\title{
The Effect of U-Shaped Patterns to Nonlinear Properties of Heart Rate Variability
}

\author{
Mateusz Soliński ${ }^{1}$, Rafał Baranowski ${ }^{2}$, Beata Graff $^{3}$, Jan Żebrowski ${ }^{1}$ \\ ${ }^{1}$ Faculty of Physics, Warsaw University of Technology, Warsaw, Poland \\ ${ }^{2}$ Department of Cardiac Rehabilitation and Noninvasive Electrocardiology, Institute of Cardiology, \\ Warsaw, Poland \\ ${ }^{3}$ Department of Hypertension and Diabetology, Medical University of Gdańsk, Gdańsk, Poland
}

\begin{abstract}
$U$-shaped patterns are acceleration-deceleration events characterized by high persistency of $R R$ time intervals. Percentage contribution of $U$-shaped patterns to whole night-time series is small (in average 3\%). However, the occurrence of $U$-shaped patterns affects to the properties of heart rate variability (HRV) significantly. The aim of this study is to evaluate its effect to nonlinear properties of HRV considering the ageing.

Night-time 63 RR interval series from healthy subjects

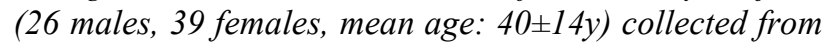
the Institute of Cardiology and the Medical University of Gdańsk were analysed. Nonlinear properties of HRV were described by using DFA, Multiscale Multifractal Analysis (MMA) and Sample entropy.

We found significant differences of Sample entropy $(p<0.01)$ and DFA $\alpha_{2}(p<0.01)$ between the signals with a small $(<=20,32 \mathrm{pts})$ and a large ( $>20,33 \mathrm{pts})$ number of $U$-shaped patterns. In turn, we observed significant difference of DFA $\alpha_{1}$ between the patients $<39 y$ (32 pts) and $>=39 y$ (33 pts) $(p<0.01)$. We also found the $U$ shaped patterns as the factor modulating the values of scaling exponents for wide range of scales.

The effect of the U-shaped patterns on HRV appears to be much stronger than expected judging by their total length compared to the length of the signal.
\end{abstract}

\section{Introduction}

Most of mathematical models of heart rate variability (HRV) simulate the fluctuation of RR time intervals durations using stochastic processes such as: 1/f noise [1], bimodal cascade [2], invert Fourier transform with shuffled phase [3], combination of random variables from exponential and power law distribution [4]. Some of models consider additional short 'bursts' simulating ectopic beats, movements or other events. However, RR time interval series contain also another characteristic and repetitive (from signal to signal) structure that we called
'U-shaped patterns'. It is defined as accelerationdeceleration event where acceleration part is longer than that of deceleration. The duration of this event is within $20-40$ seconds and its amplitude is smaller than $15 \%$ of the mean RR interval (at night). The example of U-shaped pattern is showed in Figure 1.

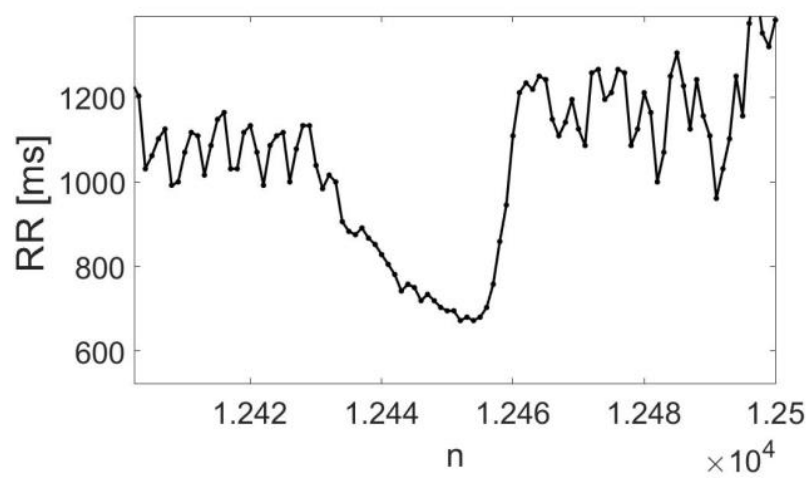

Figure 1. Example of U-shaped pattern.

Preliminary studies about the characterization of U-shaped patterns on healthy individuals showed that there is a moderate correlation between the number of $\mathrm{U}$-shaped patterns at night and the age $(\mathrm{r}=-0.39)$. Moreover, significant influence of U-shaped patterns (evaluated by comparison analysis between original data and the RR time interval series with artificially removed U-shaped patterns) on HRV parameters such as STD, low and very low frequency components, Shannon Entropy, DFA scaling exponents and mean $\mathrm{h}(\mathrm{q}, \mathrm{s})$ (Multiscale Multifractal Analysis) was observed [5,6]. Taking into account these findings the aim of this study is to evaluate the effect of U-shaped patterns to nonlinear properties of HRV considering the ageing.

\section{Data}


RR time-interval series were extracted from anonymous $24 \mathrm{~h}$ Holter ECG databases of the Institute of Cardiology (Warsaw, Poland) and the Medical University of Gdańsk (Gdańsk, Poland). Data were obtained from 65 healthy individuals, 39 females, 37.5(11.3) years old and 26 males, 41.7(16.5) years old, without organic heart disease. Only night-time parts (with constant number of samples equaled 25,000 ) of RR time interval series were used for the analysis.

\section{Methods}

\subsection{Nonlinear HRV methods}

The following non-linear method were used: a) Sample and Shannon entropy, b) Detrended Fluctuation Analysis (DFA) by calculating $\alpha_{1}(\mathrm{n}=\langle 4-16\rangle$ for evaluating short-time correlations) and $\alpha_{2}(\mathrm{n}=\langle 16-48\rangle$ for evaluating time-time correlations) scaling components and c) mean Hurst h(q,s) exponent from MMA.

\subsection{Comparison analysis}

The comparison analysis was performed to investigate the differences between the groups of RR time interval series according to the age and the number of U-shaped patterns. Firstly, HRV non-linear parameters were calculated in a group of signals with $<20$ U-shaped patterns (32 subjects) and >= $20 \mathrm{U}$-shaped patterns (33 subjects), where 20 is a median value of the number of U-shaped patterns in whole database. Secondly, the calculation was repeated in groups of the RR timeinterval series obtained from the individuals <39 years and $>=39$ years, where 39 years is the median value for whole database.

Percentage differences of the calculated parameter values between defined groups were evaluated using statistical tests: t-Student or Mann-Whitney tests (depends on normality of the distribution as checked using the Kolmogorov-Smirnov test). All calculations were performed using MATLAB 2018b and R Studio (v. 1.1.456) with $\mathrm{R}$ version: 3.5 .1 .

\section{4. $\quad$ Results}

Calculated values and the percentage differences of the HRV parameters values between the groups determined by the number of U-shaped patterns and the are shown in Tables 1 and 2 .

Table 1. Mean values and percentage differences of calculated HRV parameters in two groups of RR time interval series determined according to the number of U-shaped patterns in a signal.

\begin{tabular}{|c|c|c|c|}
\hline \multirow[b]{2}{*}{$\mathrm{N}=65$} & \multicolumn{2}{|c|}{$\begin{array}{c}\text { Number of U-shaped } \\
\text { patterns: }\end{array}$} & \\
\hline & $<20$ & $>=20$ & \\
\hline & $\begin{array}{l}\text { Mean } \\
\text { (STD) }\end{array}$ & $\begin{array}{l}\text { Mean } \\
(\text { STD })\end{array}$ & $\begin{array}{l}\text { Percentage } \\
\text { Difference }\end{array}$ \\
\hline $\begin{array}{l}\text { Number of } \\
\text { U-shaped } \\
\text { patterns }\end{array}$ & $11.9(5.6)$ & $29.8(6.6)$ & $-151.2 \% *$ \\
\hline Age & $43.3(13.1)$ & $36.5(14.7)$ & $15.8 \% *$ \\
\hline $\begin{array}{l}\text { Shannon } \\
\text { entropy }\end{array}$ & $2.67(0.16)$ & $2.73(0.05)$ & $-2.3 \% *$ \\
\hline Sample entropy & $1.06(0.29)$ & $1.23(0.02)$ & $-16.1 \% *$ \\
\hline$\alpha_{1}(\mathrm{DFA})$ & $1.14(0.22)$ & $1.12(0.18)$ & $1.5 \%$ \\
\hline$\alpha_{2}(\mathrm{DFA})$ & $0.98(0.12)$ & $1.06(0.07)$ & $-7.4 \% *$ \\
\hline Mean h(q,s) & $0.88(0.08)$ & $0.86(0.05)$ & $2.8 \%$ \\
\hline
\end{tabular}

Table 2. Mean values and percentage differences of calculated HRV parameters in two groups of RR time interval series determined according to the age of the subject.

\begin{tabular}{lccc}
\cline { 2 - 3 } \multicolumn{1}{c}{$\mathrm{N}=65$} & \multicolumn{2}{c}{ Age of the subject: } & \\
\cline { 2 - 3 } & $\begin{array}{c}\text { Mean } \\
\text { (STD) }\end{array}$ & $\begin{array}{c}\text { Mean } \\
\text { (STD) }\end{array}$ & $\begin{array}{c}\text { Percentage } \\
\text { Difference }\end{array}$ \\
\hline $\begin{array}{l}\text { Number of } \\
\text { U-shaped }\end{array}$ & $23.2(10.5)$ & $18.2(10.9)$ & $21.8 \% *$ \\
patterns & & & \\
Age & $28.6(7.4)$ & $51.7(9.0)$ & $-80.6 \% *$ \\
Shannon & $2.69(0.16)$ & $2.72(0.06)$ & $-1.0 \%$ \\
entropy & $1.18(0.28)$ & $1.11(0.24)$ & $5.8 \%$ \\
Sample entropy & $1.06(0.21)$ & $1.21(0.16)$ & $\mathbf{- 1 4 . 4 \% *}$ \\
$\alpha_{1}(\mathrm{DFA})$ & $1.01(0.10)$ & $1.03(0.11)$ & $-1.2 \%$ \\
$\alpha_{2}(\mathrm{DFA})$ & $0.86(0.06)$ & $0.87(0.08)$ & $-1.4 \%$ \\
Mean h(q,s) & & & \\
\hline \multicolumn{1}{c}{ * p $<0.05$} & &
\end{tabular}

\section{Discussion}

The results showed that there are significant differences of Shannon and Sample entropy values between the groups divided according to the number of U-shaped patterns. Taking into account these parameters, the values of entropies are higher in comparison to the group with smaller number of U-shaped patterns. 

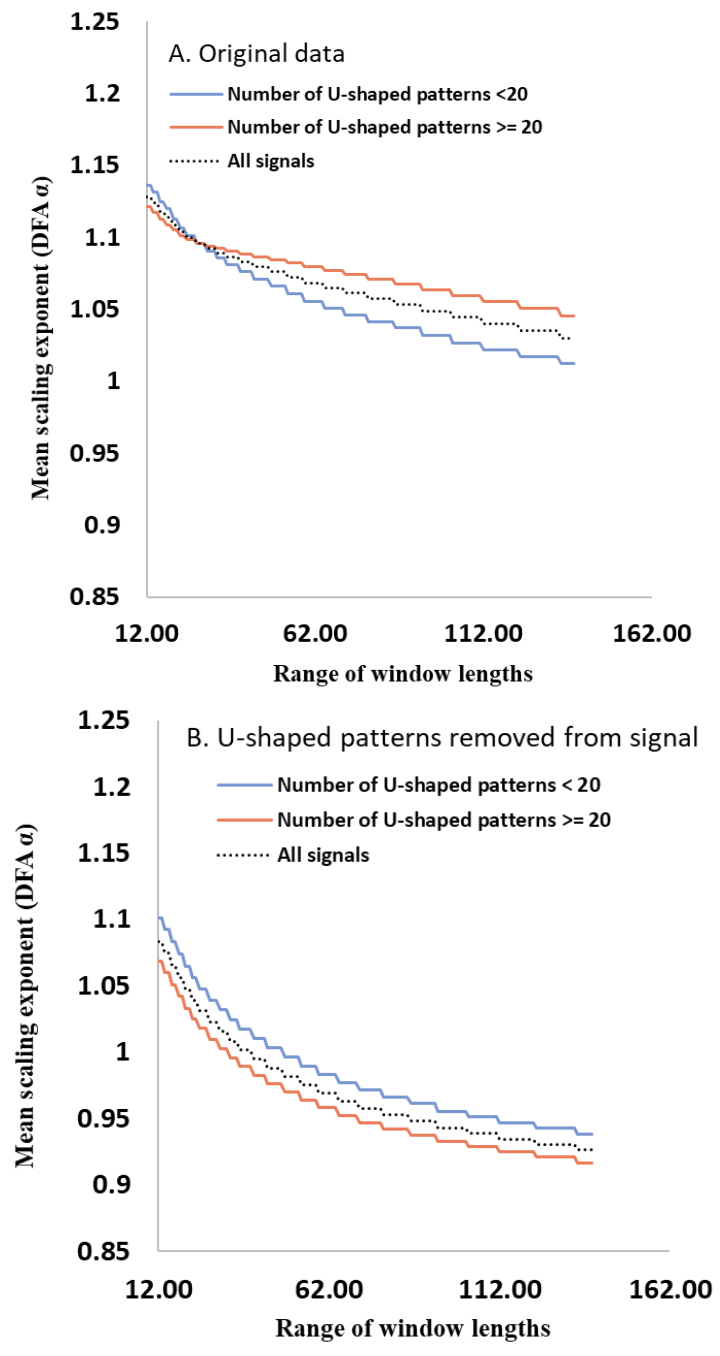

Figure 2. The values of mean scaling exponent calculated for different window lengths in two groups divided according to the number of U-shaped patterns.

Calculation of scaling exponents (DFA) showed that there is significant percentage difference of $\alpha_{2}$ between the groups with small $(<39)$ and large $(>=39)$ number of $\mathrm{U}$-shaped patterns, but there is no significant percentage difference of $\alpha_{1}$ between these groups. The opposite results are observed for the groups divided according to the age - scaling exponent $\alpha_{1}$ was significant higher in the group of the subjects $>39$ y.o. in comparison to the subjects $>=39$ y.o., while there is no significant difference for $\alpha_{2}$ scaling exponent. These findings suggest that U-shaped patterns and ageing are responsible for the modulation of the different scales of autocorrelation and so of the fractal properties. Taking into account observed differences, the analysis of continuous spectrum of scaling exponents $\alpha$ was performed according to the following rule: $\alpha_{<4-x>}$ is calculated by the fitting the line to the values of fluctuation function in a range $\langle 4, \mathrm{x}\rangle$, where $\mathrm{x}$ is the number between 16 and 116 . Hence, the larger $\mathrm{x}$, the wider scales of correlation are analyzed. The values of scaling exponents for different lengths of the windows of samples of fluctuation function, calculated separately for the groups divided according to the number of $\mathrm{U}$-shaped patterns and the age are shown in figures 2-4. Moreover, the figures show the same calculations performed for RR time interval series where U-shaped patterns were removed from the signal.
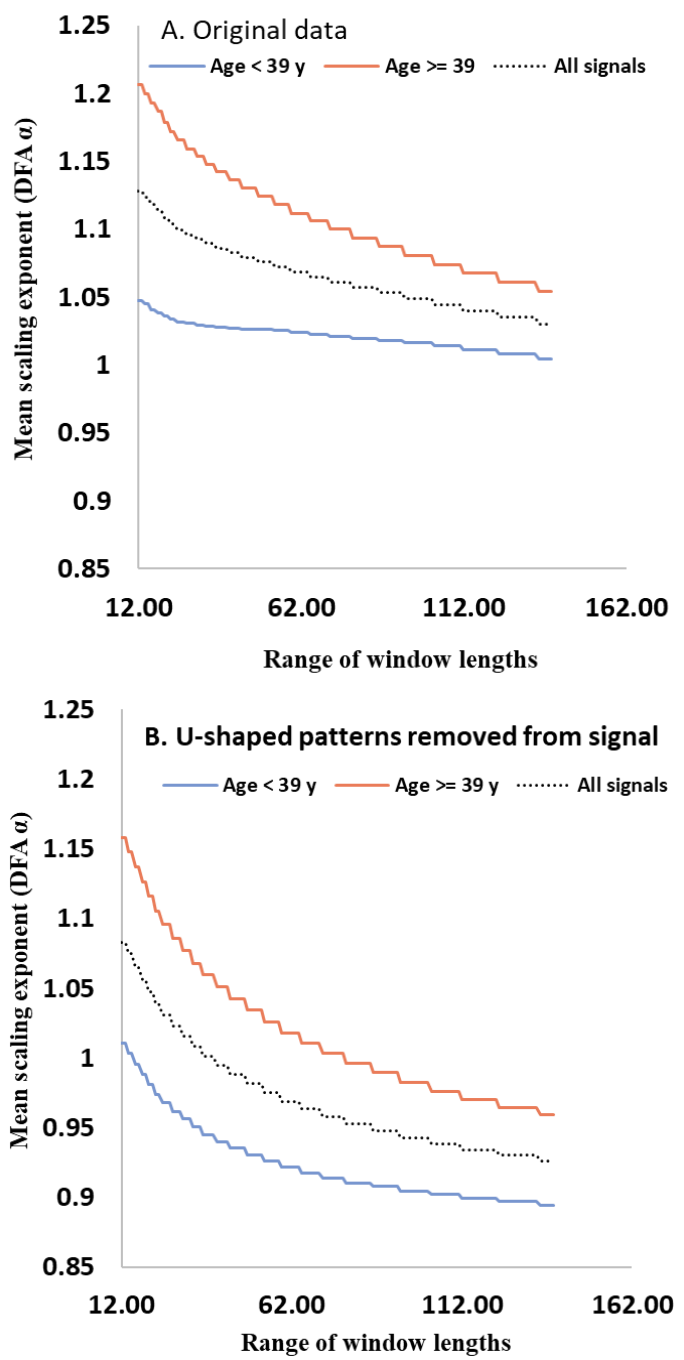

Figure 3. The values of mean scaling exponent calculated for different window lengths in two groups divided according to the age of the subjects. 

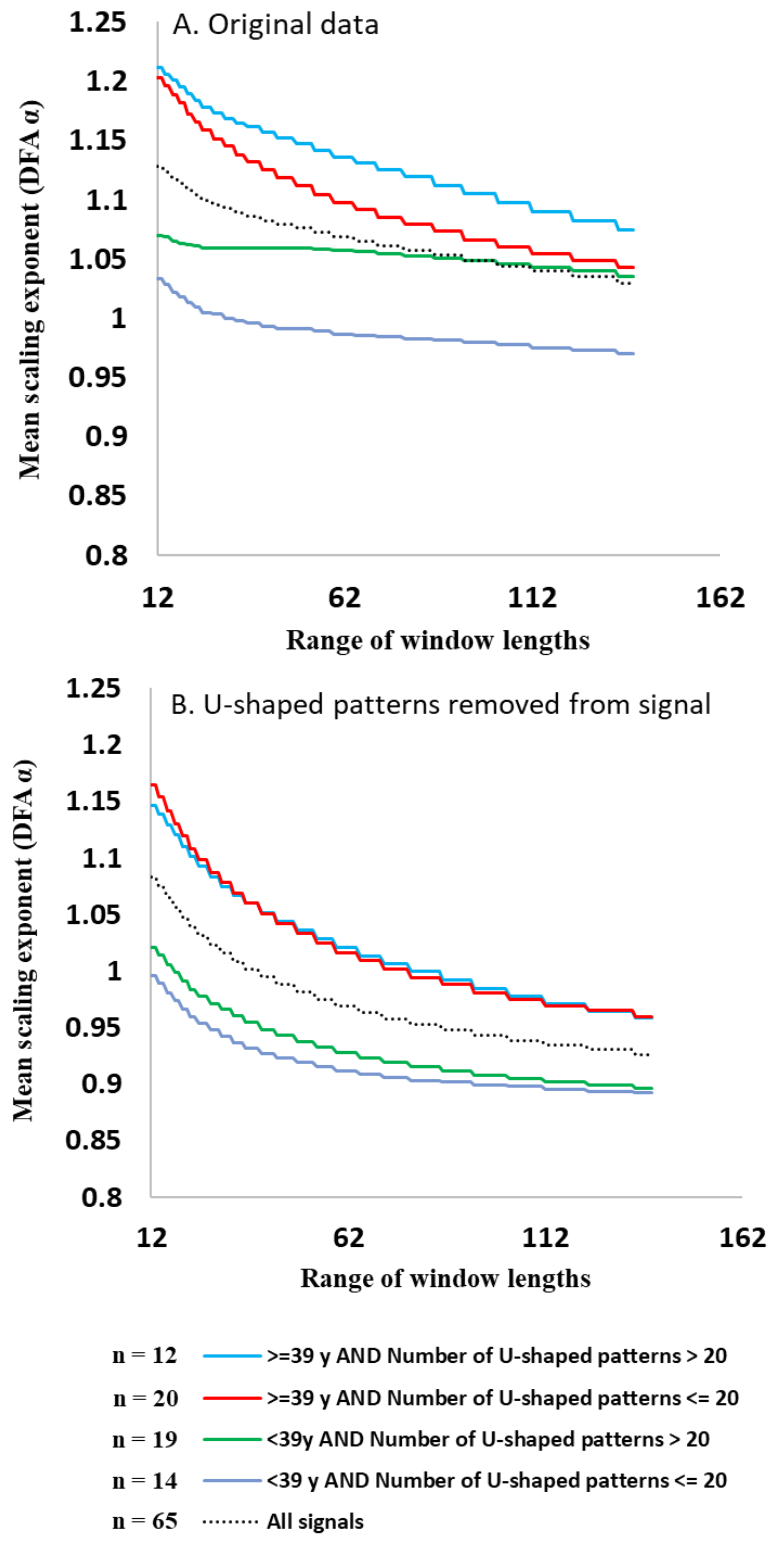

Figure 4. The values of mean scaling exponent calculated for different window lengths in four groups divided according to the number of $\mathrm{U}$-shaped patterns and the age of the subjects.

Different changes of scaling exponents with the length of the window of samples of fluctuation function values are observed depending on how the base is divided. The values of $\alpha$ exponents in a group of the signals with small and large numbers of U-shaped patterns are very similar for small window lengths (or even equal for the length about 16 samples, see Figure 2). The difference between compared groups increases with the length of the window. In turn, the difference of $\alpha$ exponents between the group of younger and older subjects (see Figure 3) is the largest for small window lengths. Figure 4 shows the combination of the effects of these two factors. Moreover, the values of the $\alpha$ exponent calculated from the RR time intervals with removed U-shaped patterns (Figures $2 b, 3 b$ and $4 \mathrm{~b}$ ) change in other way with the length of the window in comparison to the original data. The differences between compared groups remain but the curves observed in a whole range of window lengths are parallel which suggest the reduction of the multifractal properties of RR time interval series.

\section{Conclusions}

Modulation of fractal properties caused by U-shaped patterns and ageing are observed in different scales: ageing affects short-time correlations, while U-shaped patterns influence on long-term correlations.

Removing U-shaped patterns from the RR time intervals series changes (,reduce') the fractal properties in wide range of scales. This finding should be taking into account in the analysis of the 'variability' of heart rate variability - either between groups of patients or between the data obtained from a single patient in different days. This statement reinforces the findings of the Yazdani study, which showed that the number of U-shaped patterns varies from night to night in individual patients [7].

\section{References}

[1] Ivanov PC, Nunes Amaral LA, Goldberger AL, Havlin S, Rosenblum MG, Stanley HE, Struzik ZR, "From 1/f noise to multifractal cascades in heartbeat dynamics", Chaos, 2001, 11(3):641-52.

[2] Lin DC, Hughson RL, "Modeling heart rate variability in healthy humans: a turbulence analogy", Phys. Rev. Lett. 2001, 86:1650.

[3] McSharry PE, Clifford GD, "A statistical model of the sleepwake dynamics of the cardiac rhythm", Comput. Cardiol. 2005, 25:591-594.

[4] Kantelhardt JW, Havlin S, Ivanov PC, "Modeling transient correlations in heartbeat dynamics during sleep", Europhys. Lett. 2003, 62:147-153.

[5] Soliński M, Gierałtowski J, Żebrowski J, „Modeling heart rate variability including the effect of sleep stages", Chaos 2016, 26:023101.

[6] Soliński M, Gierałtowski J, Żebrowski J, Kuklik P, „Influence of U-shape accelerations of heart rate on very low frequency band and heart rate multifractality". Comput. Cardiol. 2017, 44.

[7] Yazdani S, Cherqui A, Bourdillon N, Millet G, Vesin JM. "Analysis of U-Shape patterns in RR-Interval time series during sleep", Comput. Cardiol. 2018, 45.

Address for correspondence:

Mateusz Soliński, Koszykowa 75 St. Warsaw 00-662 Poland mat.solinski@gmail.com, solinski@if.pw.edu.pl 